Trauma Berufskrankh 2008 · 10[Suppl 1]:53-58 DOI 10.1007/s10039-007-1218-3

Online publiziert: 21. März 2007

๑) Springer Medizin Verlag 2007

\author{
W. Grechenig ${ }^{1}$ W. Pichler ${ }^{1} \cdot$ A. Weiglein ${ }^{2}$ \\ ${ }^{1}$ Universitätsklinik für Unfallchirurgie, LKH-Universitätsklinikum, Graz \\ ${ }^{2}$ Anatomisches Institut, Graz
}

\section{Frakturen des proximalen Humerus}

\section{Mögliche Ursachen von Fehlschlägen bei Osteosynthesen}

Von ihr zweigt die A. ascendens ab, die die lange Bizepssehne unterkreuzt, hier einige Äste an das Tuberculum minus abgibt und dann entlang und lateral des Sulcus bicipitalis nach proximal verläuft. Anschließend tritt sie auf Höhe des Tuberculum majus, als A. arcuata, in den $\mathrm{Hu}-$ meruskopf ein. Sie versorgt nahezu $2 / 3$ der Epiphyse. Nur einige Anteile der dorsalen Bereiche des Tuberculum majus und des dorsalen Kopfanteils werden von $\operatorname{der}$ A. circumflexa humeri posterior (- Abb. 1) versorgt. Die Äste der beiden zirkumflexen Arterien sind die einzigen, die in den Kopf eintreten.

Keine Anastomosen scheinen zwischen A. circumflexa anterior und A. circumflexa posterior, der A. thoracoacromialis und der A. suprascapularis zu bestehen, wie Gefäßinjektionen von Gerber et al. [4] bestätigten. Die A. arcuata scheint eine Endarterie zu sein und sollte in keinem Fall ligiert werden [4].

Des Weiteren spielen in der Fraktursituation Äste der A. circumflexa anterior und A. circumflexa posterior eine Rolle, die die mediale Gelenkkapsel und den medialen Kopfanteil versorgen und als ventromediale und dorsomediale Arterien bezeichnet werden [21]. Frakturen, bei denen es nicht zu einer Unterbrechung der Gelenkkapsel an der medioinferioren Zirkumferenz des Humeruskopfs kommt, sind weniger gefährdet, eine komplette avaskuläre Kopfnekrose nach sich zu ziehen.

Aus diesem Grund ist es unerlässlich, als Chirurg bei der Versorgung derartiger Frakturen die Gefäßsituation des $\mathrm{Hu}$ meruskopfs zu verstehen und durch eine exakte, präoperative Planung einen iatrogenen Schaden so gering als möglich
- das Erreichen einer schmerzfreien adäquaten Funktion [13].

Aufgrund des steigenden Durchschnittsalters der Bevölkerung ist mit einer stetigen Zunahme von Frakturen zu rechnen [15]. Nach dem 50. Lebensjahr ist ein sprunghafter Anstieg der Verletzungshäufigkeit zu verzeichnen. Benachteiligt durch die Involutionsosteoporose sind in 80\% der Fälle Frauen betroffen $[7,11]$.

\section{Gefäßanatomie des proximalen Humerus}

Die hauptversorgende Arterie ist die A. circumflexa humeri anterior (• Abb. 1).
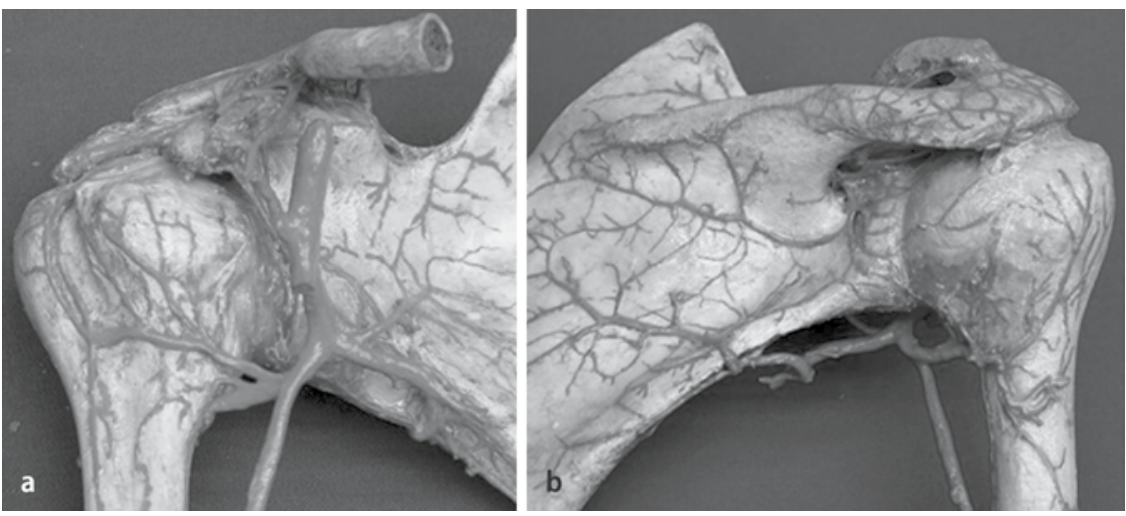

Abb. $1 \Delta$ Gefäßanatomie des proximalen Humerus, a A. circumflexa humeri anterior, b A. circumflexa humeri posterior 


\section{Die verletzte Schulter}

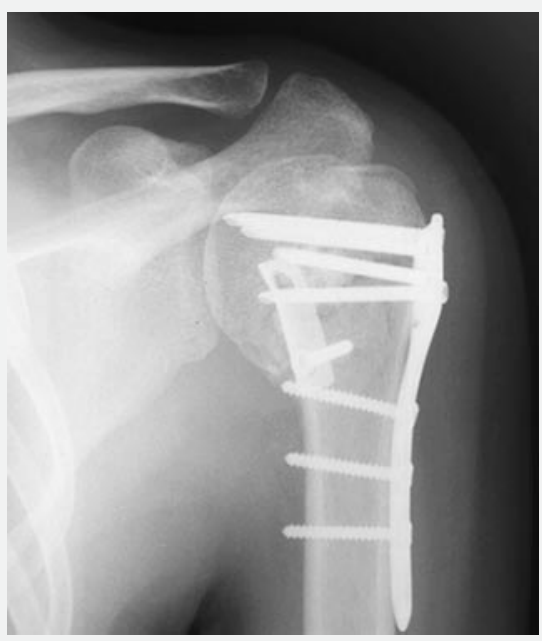

Abb. $2 \Delta$ Postoperatives Röntgen, winkelstabile Humerusplatte (LCP-PHP) in korrekter Position, medial zusätzlich Kleinfragmentplatte, direkte Manipulation und Präparation medial führen in jedem Fall zur Gefährdung der Durchblutungssituation des Humeruskopfs und Störung der Frakturheilung

zu halten. So wurden bei Osteosynthesen anfänglich Humeruskopfnekroseraten von $12-76 \%$ beschrieben [12, 14], welche allein durch eine schonende Operationstechnik unter Verwendung normaler Platten (T-Platte, Kleeblattplatte) deutlich auf $0-5 \%$ gesenkt werden konnten [11, 25, 26]. Bei offenen Osteosynthesen werden somit Repositionsmanöver des Kalottenfragments durch lateral zwischen den Tubercula eingebrachte Raspatorien oder Ähnliches durchgeführt, die in jedem Fall die mediale Kopf-Hals-Region und auch die ventromedialen und ventrodorsalen Anteil nicht tangieren (- Abb. 2). Fragmente sollten medial nicht mit Repositionszangen gefasst werden, und die indirekte Reposition sollte immer durch Manipulation am Arm und Repositionstechnik mit den Implantaten (z. B. Reponieren an die Platte) durchgeführt werden.

\section{Humerusfrakturklassifikation}

\section{Vorhandene Klassifikationssysteme}

Die am häufigsten verwendete Einteilung ist die von Neer, welche die Hauptsegmente des Humeruskopfs berücksichtigt, wobei die Fehlstellung der einzelnen Fragmente eine bedeutsame Rolle spielt, um in eine der Kategorien zu fallen. Ei-

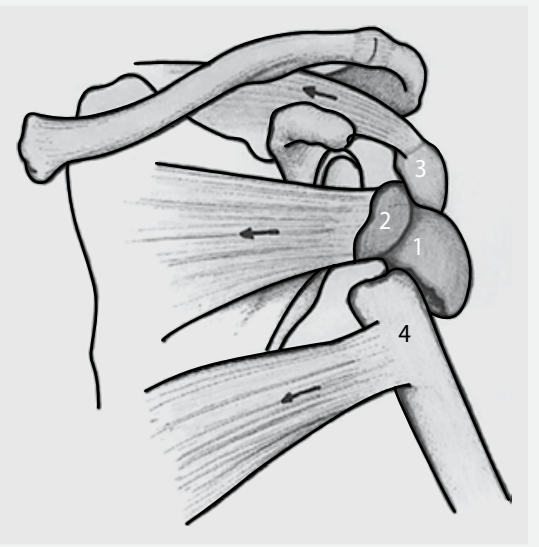

Abb. $3 \Delta$ Muskelzug von M. supraspinatus, M. subscapularis und M. pectoralis major mit typischer Dislokationstendenz der Fragmente

ne Dislokation jedes einzelnen Fragments $>10 \mathrm{~mm}$ und eine Achsenfehlstellung $>45^{\circ}$ ergeben automatisch eine höhere Frakturkategorie und damit eine schlechtere Prognose [17].

Die 2. Frakturnomenklatur, inauguriert von M. Müller, wurde 1984 von der SICOT empfohlen und entspricht der ABC-Klassifikation, die auch in anderen Extremitätenregionen breite Anwendung findet. Sie wurde 1990 modifiziert $[9,16,24]$.

\section{Sinn der Fraktureinteilung}

Eine exakte präoperative Klassifikation des Bruchs unter Würdigung der so genannten Persönlichkeit der Fraktur ist nicht nur notwendig, um eine wissenschaftliche Auswertung vergleichbarer Frakturtypen $\mathrm{zu}$ ermöglichen, sondern auch die wichtigste Voraussetzung für eine exakte präoperative Planung, möglichst schonende Reposition und Osteosynthese.

Durch die Vielzahl der verschiedenen Frakturtypen besteht aber auch das Risiko einer Fehlklassifizierung, die von mehreren Autoren beschrieben wurde, wobei die Möglichkeiten sowohl eines Inter- als auch eines Intraobserverirrtums bestehen $[10,22,23]$.

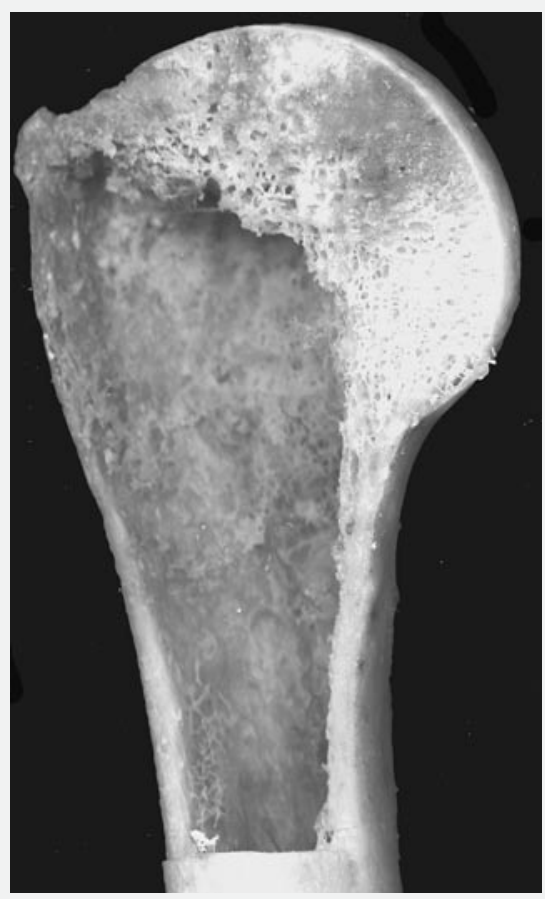

Abb. $4 \Delta$ Anatomisches Schnittpräparat des proximalen Humerus

\section{Klassifikationskriterien}

In jedem Fall sollten Röntgenbilder in 2 unterschiedlichen Ebenen und von ausreichend guter Bildqualität vorliegen. Bei komplexeren Frakturen ist die Computertomographie mit der Möglichkeit der zwei- und besonders auch der dreidimensionalen Rekonstruktion unerlässlich. Neben der exakten Beurteilung der Fraktursituation können auch die Größe des Kalottenfragments und mehrfach frakturierte Tuberculaanteile beurteilt und ein erster Eindruck über die Knochenqualität (Osteoporose, Zysten) gewonnen werden. Durch die exakte Analyse und Rekonstruktion kann beurteilt werden, ob die Größe und Knochenqualität des Kalottenfragments ausreichen, um mit Schrauben genügenden Halt zu finden.

Bei der Trauma- und Frakturanalyse müssen die durch den Muskelzug verursachten Dislokationen des Tuberculum majus und minus sowie die Stellung des Kalottenfragments analysiert werden (- Abb. 3), um eine schonende perkutane bzw. offene Reposition und Fixation zu ermöglichen. Der mediale Knochensporn, die Verschiebung des Schaftfragments in Relation zum Kalottenfragment, die Höhe des Tuberculum majus und eine evtl. Antekurvation müssen berücksichtigt wer- 
den. Bei Verschiebungen zwischen Schaft und Kopf über 6-9 mm muss von einer Unterbrechung der Blutversorgung für den Kopf ausgegangen werden [18]. Des Weiteren ist die Verschiebung des Kalottenfragments nach medial günstiger einzustufen als eine nach lateral.

Bei komplexen 4-Fragment-Frakturen des älteren Patienten kann auch eine präoperative MRT-Untersuchung zur Beurteilung der Rotatorenmanschette und daraus resultierender weiterer Operationsplanung (Typ der Endoprothese) notwendig sein.

\section{Geometrie des proximalen Humerus}

Die Extremitas proximalis humeri umfasst das Caput humeri und die zwischen Collum anatomicum und Collum chirurgicum liegenden Tubercula majus und minus, welche durch den Sulcus intertubercularis voneinander getrennt sind (- Abb. 4) $[2,6,19,20]$.

Das Caput humeri entspricht in $88,2 \%$ der Fälle einer sphärischen Kugel mit einer Durchmesserabweichung $<1 \mathrm{~mm}$, wobei etwa $2 / 3$ dieser Kugel mit Knorpel überzogen sind. Der individuelle Durchmesser variiert aber stark von 37,1-56,9 mm (Mittelwert: 46,2 mm).

Große Variationen bestehen auch bei der relativen Lage des Caput humeri zum Humerusschaft. Einerseits ist der Humeruskopf, ähnlich wie der Hüftkopf, aber mit einem wesentlich kürzeren Hals, im Sinne einer Varisierung nach medial geknickt (• Abb. 5, 6). Der dem FemurCCD-Winkel entsprechende Neigungswinkel (Inklinationswinkel) variiert individuell zwischen $114^{\circ}$ und $147^{\circ}$ (Mittelwert: $130^{\circ}$ ), wobei $95 \%$ im Bereich von $134-145^{\circ}$ liegen. Die Seitendifferenz bei einem Individuum ist allerdings sehr gering und liegt im Mittel bei 2,1 ${ }^{\circ}$. Andererseits ist der Humeruskopf gegenüber der Interepikondylarlinie des distalen Humerus nach dorsal zur Cavitas glenoidalis der Skapula gedreht. Der entsprechende Torsionswinkel (Retroversionswinkel) unterliegt extremen individuellen Schwankungen und reicht von $6,5-47,5^{\circ}$ (Mittelwert $\left.17,9^{\circ}\right)$.

Der Humerusschaft ist leicht nach ventral konvex gebogen, die Kompak-
Trauma Berufskrankh 2008 · 10[Suppl 1]:53-58 DOI 10.1007/s10039-007-1218-3

(c) Springer Medizin Verlag 2007

W. Grechenig · W. Pichler · A. Weiglein

Frakturen des proximalen Humerus.

Mögliche Ursachen von Fehlschlägen bei Osteosynthesen

\section{Zusammenfassung}

Bei 4-5\% aller Frakturen handelt es sich um proximale Humerusfrakturen, begünstigend wirkt v. a. die Osteoporose. Aus diesem Grund ist unter Berücksichtigung der derzeitigen demografischen Entwicklung mit einer Zunahme dieser Verletzungen zu rechnen. In den meisten Fällen werden mit einer konservativen Behandlung mit Ruhigstellung und Schulterverband gute Ergebnisse erreicht, in etwa 30\% der Fälle ist jedoch eine operative Intervention erforderlich. Aufgrund der komplexen Anatomie und Gefäßversorgung ist der Eingriff schwierig. Komplizierend hinzu kommen das meist höhere Lebensalters der Patienten und damit deren häufig einge-

schränkte Knochenqualität. Um ein möglichst gutes postoperatives Ergebnis erreichen zu können, sind eine exakte Einteilung der Fraktur, eine detaillierte präoperative Planung, ausreichende Kenntnisse über die Anatomie und Gefäßversorgung der Oberarmregion sowie die möglichen chirurgischen Techniken und eine individuelle Nachbehandlung unabdingbar.

\section{Schlüsselwörter}

Proximale Humerusfraktur · Ruhigstellung .

Operative Intervention - Klassifikation .

Gefäßversorgung

\section{Fractures of the proximal humerus. Possible reasons for failed osteosynthesis}

\section{Abstract}

Fractures of the proximal humerus account for $4-5 \%$ of all fractures, with the chief predisposing factor being osteoporosis. This means that in view of the present demographic developments we have to expect increasing numbers of these injuries. In most cases good reesults are achieved with a conservative treatment involving immobilization and bandaging of the shoulder, but in about $30 \%$ of cases surgery is needed. The surgical operation is difficult because of the complex anatomy and vascularitation. The difficulty is compounded by the usually quite advanced age of the patients and their corresponding- ly poor bone quality. Achievement of the best possible postoperative result demands accurate classification of the fracture, detailed preoperative planning, adequate knowledge of the anatomy and vascularization of the upper arm region and of the surgical techniques available, and an individually tailored aftertreatment.

\section{Keywords}

Proximal fracture of the humerus . Immobilization - Operative intervention . Classification · Vascularization 


\section{Die verletzte Schulter}

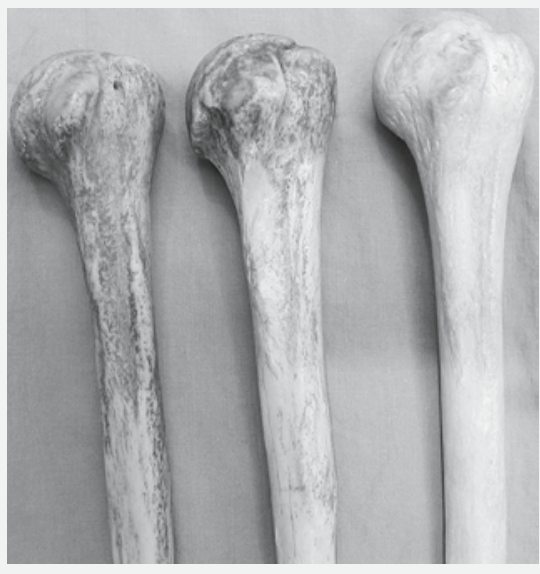

Abb. $5 \Delta$ Große Variationen zwischen Caput humeri und Humerusschaft bei verschiedenen anatomischen Präparaten

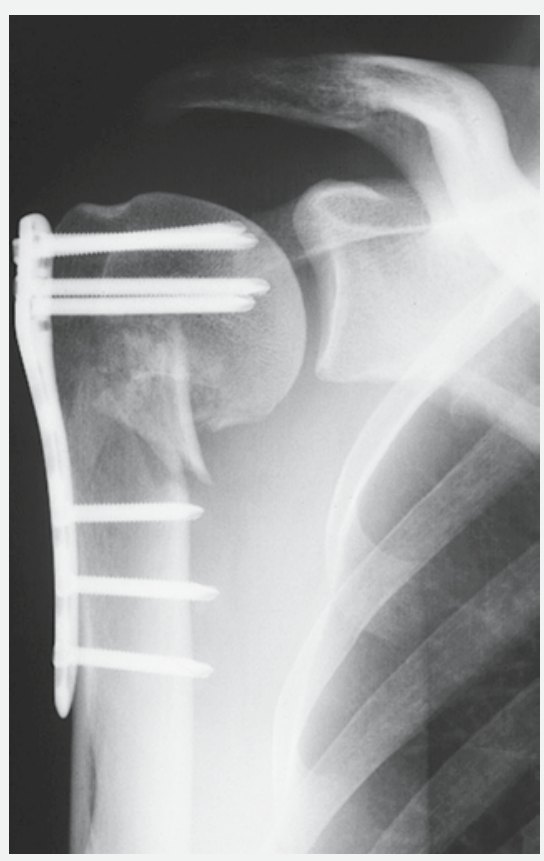

Abb. $7 \Delta$ Schlechte, ungenügende Reposition bei deutlicher Varusstellung des Kopffragments

ta umgibt mit 3-5 mm Dicke den Markraum, der entsprechend der äußeren Form des Schafts proximal annähernd zylindrisch und weit ist und nach distal hin enger und flacher wird.

Entsprechend anatomischer Untersuchungen berücksichtigen winkelstabile Implantate bei der Schraubenlage jene Kopfregionen, bei welchen die Spongiosa am besten ausgebildet und der Halt der Schrauben somit sicherer gewährleistet sind. Unter Berücksichtigung der Tatsache, dass die Konfiguration und die Schraubenrichtung dieser Platten vorge-

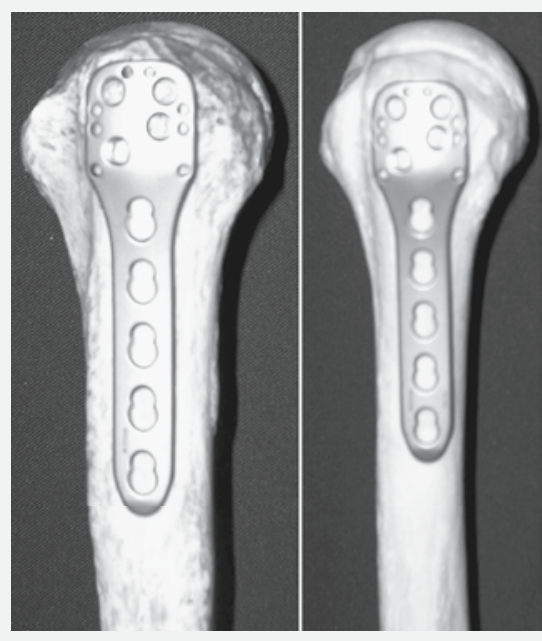

Abb. $6 \Delta$ Relation zwischen kranialem Anteil der Platte bzw. kranialem Rand des Tuberculum majus und kranialem Rand der Oberarmkopfkalotte bei Ansicht von lateral

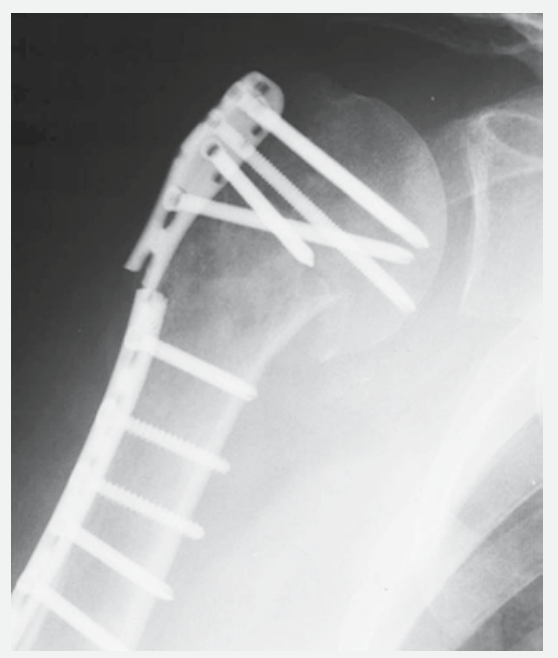

Abb. $8 \Delta$ Bei fehlender medialer Abstützung und hoher Stresskonzentration der Platte drohender Plattenbruch (Implantatversagen)

geben sind, müssen die unterschiedlichen anatomischen Konfigurationen der proximalen Oberarmregion mit berücksichtigt werden. Dies führt dazu, dass die exakte Plattenposition nicht immer einfach zu bestimmen ist und in der Fraktursituation die Geometrie der Kopf-/Halsregion (z. B. stärkerer Varus) oft nicht sicher beurteilt werden kann. Bei winkelstabilen Systemen ist ab dem Moment der Plattenfixation die Richtung der Schrauben in der Regel vorgegeben. Neuere Platten tragen diesem Umstand Rechnung und erlauben ein freies Einbringen der Schrau- ben und die winkelstabile Schraubenplattenverankerung.

\section{Operationstechnik}

Die Therapie der komplexen Frakturtypen mit 3, 4 oder mehr Fragmenten und zunehmender Dislokation gilt generell als schwierig. In der aktuellen Literatur werden unterschiedliche Behandlungskonzepte favorisiert. Das Spektrum reicht von der konservativen Therapieform über aufwändige anatomische Rekonstruktionen bis zur Endoprothetik. Der Trend zur minimalinvasiven und biologischen Osteosynthese in der Unfallchirurgie hat sich auch am Humeruskopf manifestiert $[3,11$, $18,25]$.

\section{Kirschner-Drähte}

Die alleinige Verwendung perkutan eingebrachter Kirschner-Drähte stellt nach wie vor eine Standardbehandlung bei Frakturen im Wachstumsalter dar. Bei Erwachsenen werden die Drähte vorzugsweise lateral am Humerus verblockt (z. B. Humerusblock nach Resch). Dies erhöht die Stabilität. Eine Diastase in der subkapitalen Frakturzone ist aber unbedingt $\mathrm{zu}$ vermeiden. Bei der alleinigen Verwendung von Kirschner-Drähten müssen diese, wenn sie unter der Haut versenkt werden, unbedingt umgebogen werden, um ein Wandern derselben zu vermeiden.

\section{Implantate}

Bei der Verwendung intramedullärer Implantate stehen Komplikationen bei der Einbringungsstelle (Sehnenkappe) im Vordergrund. Es ist entscheidend, dass das Implantat nicht zu weit lateral (am Sehnenansatzbereich am Tuberculum) eingebracht und am Ende der Operation der Sehnenrand evtl. exzidiert und die Sehne ordentlich vernäht werden. Sowohl bei perkutanen als auch bei offenen Techniken stellen die weitgehend anatomische Reposition des Kopf-/Halsfragments und die dadurch gewonnene Stabilität ohne Denudierung der Frakturränder den Schlüssel zum Erfolg dar. Insbesondere ist eine Varusposition des Kopfs (• Abb. 7) $\mathrm{zu}$ vermeiden, aus welcher auch eine zu weit kraniale Position der Platte resultiert. 
Abb. 9 Schwierigkeiten bei der Wahl der Schraubenlänge bei winkelstabilen Implantaten, a zu kurz gewählte Schrauben: ungenügende Verankerung in der Kopfkalotte, b zu lang gewählte Schrauben: Irritation der Cavitas glenoidalis, nachfolgend Arthrose und entsprechende Beschwerden
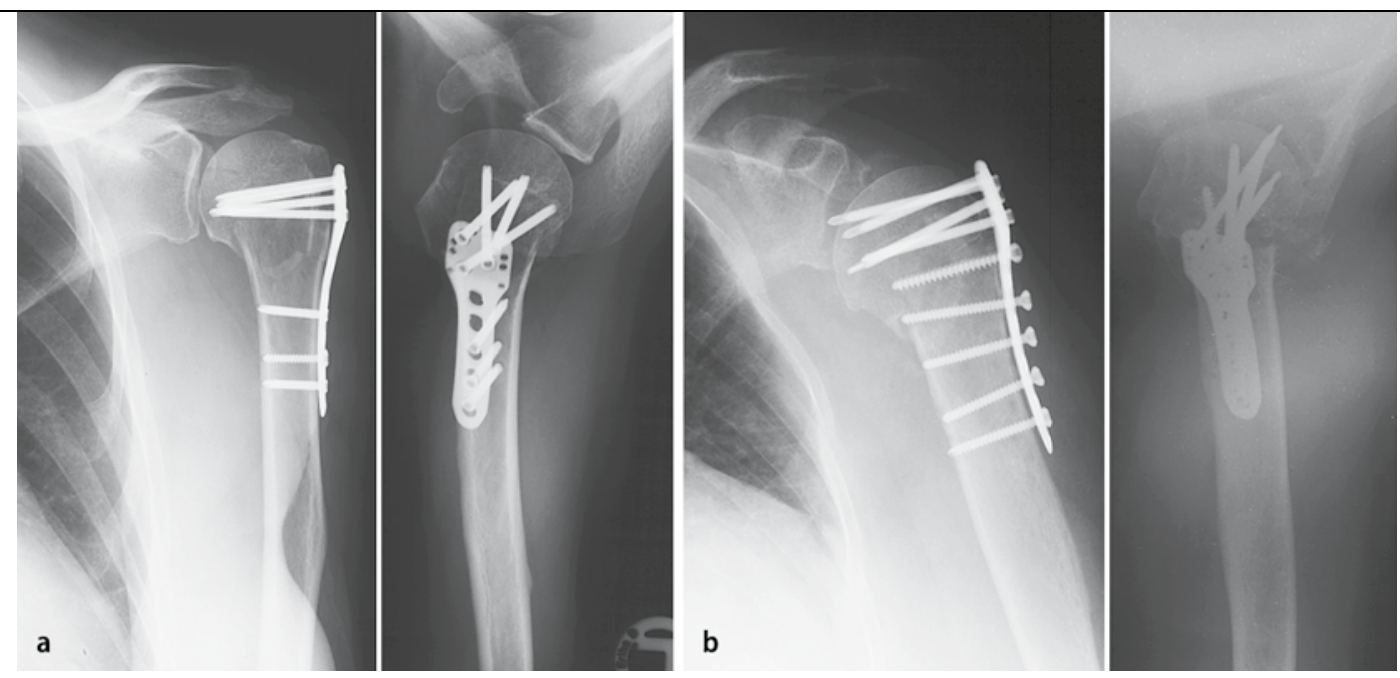

Bei fehlender medialer Abstützung kann eine zusätzliche Stabilität durch Einstauchung des Schafts in den Kopf oder durch zusätzliche Verschraubung des Tuberculum minus oder kleine ventrale Platten erreicht werden. Wenn der Schaft in das Kopffragment eingestaucht wird, ist bei Platzieren der Platte unter Verwendung konventioneller Schrauben eine Lateralisation des Schafts gegenüber dem Kopffragment zu vermeiden. Bei fehlender medialer Abstützung und hoher Stresskonzentration der Platte droht der Plattenbruch (Implantatversagen) (• Abb. 8).

Insbesondere bei der Verwendung von winkelstabilen Implantaten ist die Wahl der Schraubenlänge problematisch (- Abb. 9). Unter Beachtung des oft nur sehr schmalen Kopf-/Kalottenfragments sollten die Schrauben knapp unter der Knochenoberfläche platziert werden, ohne beim Bohren die Gegenkortikalis zu perforieren. Dies gelingt oft nur unter direkter Bildwandlerkontrolle. Der Umstand, dass selbst schneidende Schrauben an der Spitze kein Gewinde haben und die Schrauben vielfach nur in 5-mm-Abständen verfügbar sind, erklärt die häufigen Probleme bei der Wahl der richtigen Schraubenlänge.

Bei der Verwendung von Platten sollten die Tubercula durch Nähte, geführt durch den Ansatzbereich der Sehnen, an die Platte gesichert werden.

\section{Postoperative Maßnahmen}

Eine der Fraktursituation, der Knochenqualität und der Compliance des Patienten angepasste Nachbehandlung ist ebenso wichtig wie die korrekte Osteosynthese. Die entsprechenden Kräfte am proximalen Humerus bei Aktivitäten des täglichen Lebens müssen berücksichtigt werden. So treten bei Außenrotation des Arms unter Berücksichtigung des Kopfdurchmessers und des Hebelarms Kräfte von bis zu $1000 \mathrm{~N}$ auf.

\section{Fazit}

Aufgrund der komplexen Anatomie und schwierigen Gefäßversorgung und unter Berücksichtigung des meist höheren Lebensalters der Patienten mit eingeschränkter Knochenqualität stellt die chirurgische Therapie der Frakturen des proximalen Oberarms hohe Anforderungen an den Chirurgen. Mit exakter präoperativer Planung, Klassifikation des Frakturtyps mit anschließender subtiler biologischer Operationstechnik, exakter Kenntnis des Osteosyntheseverfahrens (Implantate) und falladaptierter Nachbehandlung ist in vielen Fällen ein gutes funktionelles Ergebnis zu erreichen. Bei einfachen und unverschobenen Frakturformen, aber auch bei komplexen Brüchen muss die konservative Therapie im Behandlungsalgorithmus immer mit berücksichtigt werden.

\section{Korrespondierender Autor}

\section{Prof. Dr. W. Grechenig}

Universitätsklinik für Unfallchirurgie, LKH-Universitätsklinikum Graz, Auenbruggerplatz 7a, 8036 Graz greiner-roder@klinikum-graz.at

Interessenkonflikt. Es besteht kein Interessenkonflikt. Der korrespondierende Autor versichert, dass kei- ne Verbindungen mit einer Firma, deren Produkt in dem Artikel genannt ist, oder einer Firma, die ein Konkurrenzprodukt vertreibt, bestehen. Die Präsentation des Themas ist unabhängig und die Darstellung der Inhalte produktneutral.

\section{Literatur}

1. Böhler L (1977) Oberarmbrüche. In: Böhler L (Hrsg) Technik der Knochenbruchbehandlung. Maudrich, Wien, S 644

2. Boileau P, Walch G (1997) The three-dimensional geometry of the proximal humerus. J Bone Joint Surg Br 79-B: 857-865

3. Fankhauser F, Schippinger G, Weber K et al. (2003) Cadaveric-biomechanical evaluation of bone-implant construct of proximal humerus fractures (Neer type 3). J Trauma 55: 345-349

4. Gerber C, Schneeberger AG, Vin H (1990) The arterial vascularization of the humeral head. J Bone Joint Surg Am 72: 1486-1494

5. Habermeyer P, Schweiberer $L$ (1989) Frakturen des proximalen Humerus. Orthopäde 18: 200-207

6. Hernigou P, Duparc F, Hernigou A (2002) Determining humeral retroversion with computed tomography. J Bone Joint Surg Am 84-A: 1753-1762

7. Hessmann MH, Sternstein W, Hansen M et al. (2005) Locked-plate fixation and intramedullary nailing for proximal humerus fracutres: a biomechanical evaluation. J Trauma Injury 2005: 11941201

8. Hintermann B, Trouillier HH, Schafer D (2000) Rigid internal fixation of fractures of the proximal humerus in older patients. J Bone Joint Surg Br 82: 1107-1112

9. Jaberg H, Warner J, Jakob RP (1992) Percutaneous stabilization of unstable fractures of the humerus. J Bone Joint Surg Am 74: 508-515

10. Jakob RP, Miniaci A, Anson P et al. (1991) Four part valgus impacted fractures of the proximal humerus. J Bone Joint Surg Br 73: 295-298

11. Kollig E, Kutscha-Lissberg F, Roetman B et al. (2003) Komplexe Brüche des Oberarmkopfes: welche Langzeitresultate sind zu erwarten? Barth, München, 128: 111-118

12. Kuner EH, Siebler G (1987) Luxationsfrakturen des proximalen Humerus - Ergebnisse nach operativer Behandlung. Unfallchirurgie 13: 64

13. Laing PG (1956) The arterial supply of the adult humerus. J Bone Joint Surg Am 38: 1105-1116 
14. Lill H, Lange K, Prasse-Badde J et al. (1997) Die TPlatten-Osteosynthese bei dislozierten proximalen Humerusfrakturen. Unfallchirurgie 23: 183-190

15. Lind T, Kroner K, Jensen J (1985) The epidemiology of fractures of the proximal humerus. Arch Orthop Trauma Surg 285-287

16. Moseley HF, Goldie J (1963) The arterial pattern of the rotator cuff of the shoulder. J Bone Joint Surg $\mathrm{Br}$ 45: 780-789

17. Müller ME, Nazarian S, Koch P et al. (1990) The comprehensive classification of fractures of long bones. Springer, Berlin Heidelberg New York, p 54

18. Resch H, Povacz P, Fröhlich R et al. (1997) Percutaneous fixation of three- and four-part-fractures of the proximal humerus. J Bone Joint Surg $\mathrm{Br} 79$ : 295-300

19. Roberts SN, Foley AP, Swallow HM et al. (1991) The geometry of the humeral head and the design of prostheses. J Bone Joint Surg Br 73-B: 647-650

20. Robertson DD, Yuan J, Bigliani LU et al. (2000) Three-dimensional analysis of the proximal part of the humerus: relevance to arthroplasty. J. Bone Joint Surg Am 82: 1594

21. Schweiberer L, Betz A, Eitel F et al. (1982) Bilanz der konservativen und operativen Knochenbruchbehandlung - Obere Extremität. Chirurg 54: 226233

22. SegglW, Weiglein A (1991) Die arterielle Blutversorgung des Oberarmkopfes und ihre prognostische Bedeutung bei Luxationen, Frakturen und Luxationsfrakturen des Oberarmkopfes. Acta Chir Austr [Suppl] 23: 92

23. Sidor M, Zuckermann JD, Lyon T et al. (1993) The Neer classification system for proximal humeral fractures. J Bone Joint Surg Am 75: 1745-1749

24. Speck M, Regazzoni P (1997) 4-Fragment-Frakturen des proximalen Humerus. Unfallchirurg 100: 349-353

25. Szyszkowitz R, Schippinger G (1999) Die Frakturen des proximalen Humerus. Unfallchirurg 102: 422428

26. Tingart M, Bäthis H, Bouillon B et al. (2001) Die dislozierte proximale Humerusfraktur: Gibt es gesicherte Therapiekonzepte? Chirurg 72: 1284-1291 